\title{
Humanity tested
}

\author{
The world needs mass at-home serological testing for antibodies elicited by SARS-CoV-2, and rapid and frequent \\ point-of-care testing for the presence of the virus' RNA in selected populations.
}

H ow did we end up here? Two ways. Gradually, then suddenly. Ernest Hemingway's passage is a fitting description for humanity's perception of the exponential growth of COVID-19 cases and deaths (Fig. 1). The worldwide spread of a highly infectious pathogen was only a matter of time, as long warned by many epidemiologists, public health experts, and influential and prominent voices, such as Bill Gates. Yet most of the world was unprepared for such a pandemic; in fact, most Western countries (prominently the United States $^{1}$ ) fumbled their response for weeks. Singapore, Hong Kong and Taiwan have shown the world that, to contain the propagation of severe acute respiratory syndrome coronavirus 2 (SARS-CoV-2), governments need to quickly implement aggressive testing (by detecting the viral RNA through polymerase chain reaction (PCR)), the isolation of those infected and the tracing and quarantining of their contacts, while educating their citizens about the need for physical distancing and basic public health measures (in particular, frequent hand-washing and staying at home if feeling unwell). When outbreaks are not detected and acted upon sufficiently early, drastic physical distancing - of the sort implemented by China at the end of January and maintained for months - can eventually suppress the outbreak (Fig. 1). It is however unclear whether Western countries that have implemented strict physical-distancing measures later in their infection curve will be able to gradually release such lockdowns, let alone see their outbreaks controlled.

Such non-pharmacological interventions aim to 'flatten' the infection curve by reducing the number of transmission chains and thus the virus' basic reproduction number - that is, the average number of new cases generated by a case in an immunologically naive population. In the absence of a safe and effective vaccine - which, if current efforts end up being successful, is unlikely to become widely available within the next two years non-pharmacological interventions will need to remain in place to reduce the threat of secondary outbreaks by maintaining the basic reproduction number below 1 . However, the type and degree of the interventions could be better tailored if

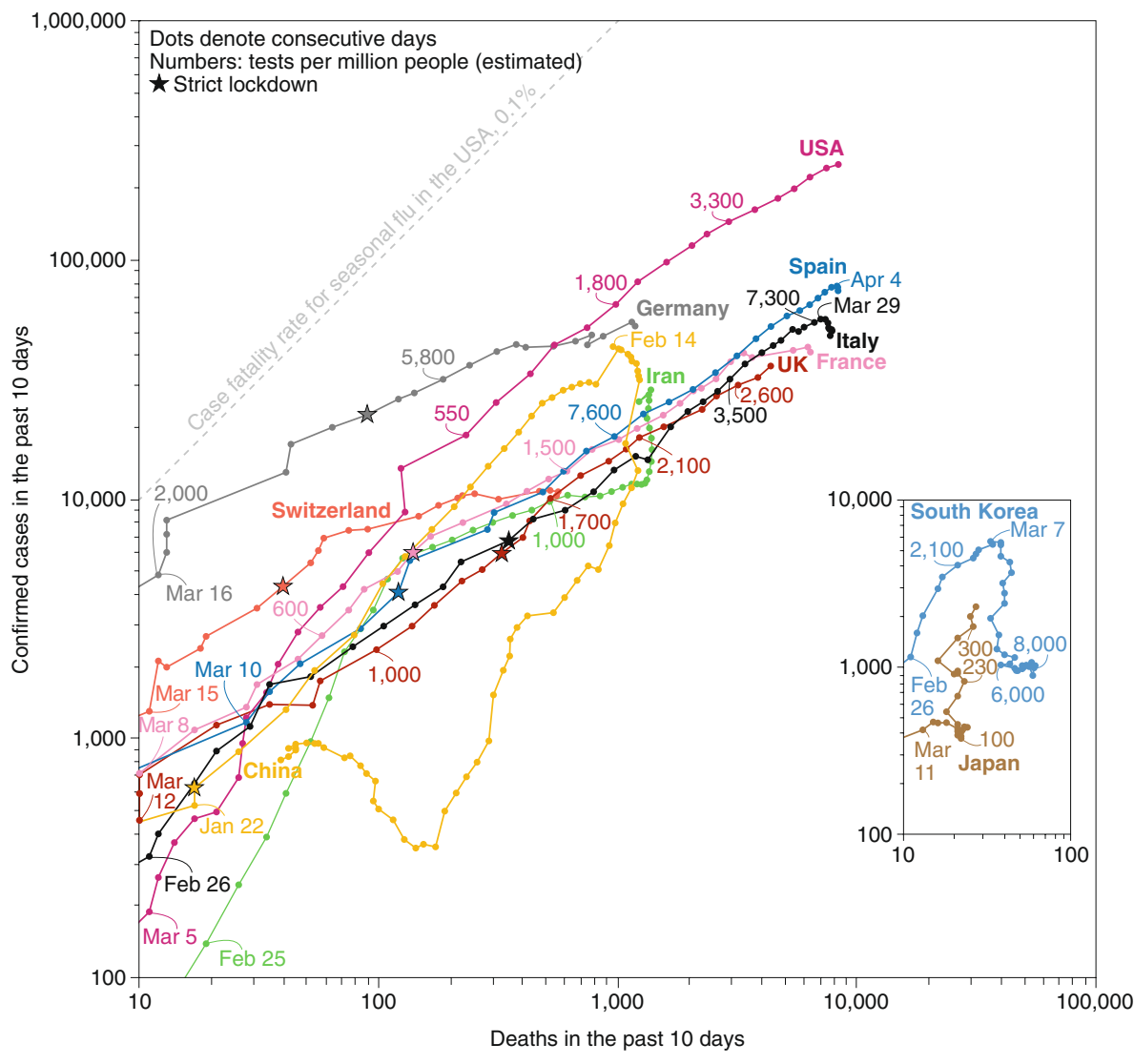

Fig. 1 | Early mass testing and early containment measures save lives. COVID-19 confirmed cases and deaths for selected countries in a 10-day window ending at each data point (successive data points on a line denote consecutive days). Numbers in colour are the estimated number of total PCR tests per million people up to the data point indicated; stars indicate when strict lockdowns were enacted. Deaths lag with respect to confirmed cases, according to the estimated two-to-three week interval ${ }^{10}$ between the onset of symptoms and death. Case fatality rates - that is, the fractions of total confirmed cases that become deaths - mostly depend on the extent of testing, on the capacity of a country's healthcare system, on its demographics and on the availability of drugs that can significantly dampen the severity of COVID-19 in those infected. Even with mass testing, the case fatality rate of COVID-19 is expected to be a multiple of that for seasonal flu in the United States (0.1\%). Countries that deployed tests for detecting SARS-CoV-2 RNA early and widely (such as South Korea), that applied contact tracing and targeted physical distancing measures for detected cases (such as South Korea and Japan), or that enacted early, strict lockdowns (such as China) are more likely to contain the disease outbreak earlier. In fact, Singapore, Hong Kong and Taiwan have contained COVID-19 outbreaks and have managed to limit COVID-19-related deaths to less than 10 (hence, these countries are not included in the figure). Data updated 6 April 2020. Individual data points can be affected by reporting errors and delays, by wilful underreporting and by location-specific definitions (and changes to them) for confirmed cases and deaths. Data sources: European Center for Disease Control and Prevention ${ }^{11}$ (cases and deaths); Our World in Data ${ }^{12}$, various government sources (tests). A regularly updated version of this graph is available ${ }^{13}$.

governments knew who are currently infected and who have been infected and recovered. For this, the world needs to see the mass deployment of serological testing for SARS-CoV-2 antibodies (which appear to be highly specific ${ }^{2}$ ), and frequent testing 
for SARS-CoV-2 RNA in those likely to be exposed to the virus (especially healthcare workers) or at a higher risk for severe respiratory disease (such as the elderly and younger individuals with relevant comorbidities).

Medical-device companies and government and research laboratories around the world have rushed to adapt and scale up nucleic acid tests (mostly employing PCR, but also CRISPR-based detection and loop-mediated isothermal amplification) to detect the virus' RNA, and government agencies are scrambling to assess them via emergency routes (such as the Emergency Use Authorization program $^{3}$ by the United States Food and Drug Administration (FDA)). Point-of-care PCR kits - based on lateral-flow technology or cartridge-based instruments for sample preparation, nucleic acid amplification and detection - also require RNA extraction from nasal or throat swabs (or both) but can speed up the time-to-result from a few hours to roughly 30 minutes $^{4}$ (and in one test, positive results can be obtained in five minutes ${ }^{5}$ ), with near-perfect sensitivity and specificity if sample acquisition and preparation and device operation are carried out appropriately by trained personnel. This limits the usefulness of these kits for at-home use, which would significantly raise the fraction of false negatives. Immunoassays incorporating monoclonal antibodies specific for SARS-CoV-2 antigens (for instance, a domain of the virus' spike protein) should be amenable to home use, yet they are more difficult to develop (the antibodies are typically obtained via the immunization of transgenic animals) and are less accurate than nucleic acid testing.

Lateral flow immunoassays (akin to the pregnancy test) and enzyme-linked immunosorbent assays to detect antibodies elicited by the virus are also being rapidly developed (mostly by Chinese companies thus far). Tens of at-home lateral-flow devices ${ }^{6}$ are already being commercialized, having obtained the European Union's CE mark or been authorized for emergency use by the FDA or the Chinese FDA.
In many of these kits, the recombinant viral antigens bind to SARS-CoV2-specific immunoglobulin $M(\operatorname{IgM})$ and immunoglobulin $\mathrm{G}$ ( $\mathrm{IgG}$ ) within $15 \mathrm{~min}$; hence, these tests can also detect early-stage infection (of which IgM levels are a marker), but at the expense of sensitivity and accuracy (which can exceed $90 \%$ and $99 \%$ for $\mathrm{IgG}^{7}$ ). The real-world performance of such serology tests, which is currently unknown, will depend on the actual prevalence of COVID-19 in the population. For example, at a $5 \%$ pre-test probability of having the disease, a test with $99 \%$ sensitivity and 95\% specificity would lead to as many true positives as false positives. Hence, before wide deployment, governments need to ensure that these finger-prick antibody tests are clinically validated ${ }^{8}$.

The world should roll out both antibody and nucleic acid tests on a wide scale. Widely available and inexpensive serological testing would help governments to tailor non-pharmacological interventions to specific locations and populations, to decide when to relax them and to permit citizens immune to the virus to help those who remain susceptible to it. Mass testing would also provide valuable data to help answer pressing unknowns: what are the infection rates across locations and populations? What fraction of the population is immune? How long does immunity last and how does it depend on age and on the severity of infection? Wider deployment of nucleic acid tests would also provide clues about the prevalence of a wider range of COVID-19 symptoms, the role of children in spreading the disease, and the epidemiological characteristics of superspreaders ${ }^{9}$ and of those who were infected and asymptomatic. Testing should be complemented by privacy-minded digital surveillance, via phone apps, aiding contact tracing and permitting lighter levels of physical distancing - as done in Singapore, South Korea and Taiwan. The downside is that any invasion of privacy via the tracking of people can last longer than necessary. De-identified and aggregated health data, such as heart rate and activity levels collected via commercial wearables, might also predict (https://detectstudy.org) the emergence and location of outbreaks.

In our globalized world, the risk of further waves of COVID-19 outbreaks, and thus of prolonged drastic economic consequences, will remain substantial as long as any outbreak anywhere remains. It is in the world's best interest that richer countries provide test kits, technical and public-health knowledge, personnel, personal protective equipment and, eventually, the necessary vaccine doses to poorer countries to assist them in their efforts to reduce and contain the spread of SARS-CoV-2. This is humanity's next test.

Published online: 8 April 2020

https://oi.org/10.1038/s41551-020-0553-6

References

1. Shear, M. D. et al. The lost month: how a failure to test blinded the U.S. to Covid-19. The New York Times https://www. nytimes.com/2020/03/28/us/testing-coronavirus-pandemic.html (2020).

2. Ju, B. et al. Preprint at https://doi.org/10.1101/2020.03.21.990770 (2020).

3. Emergency Use Authorization (U.S. Food \& Drug Administration, 2020); https://www.fda.gov/medical-devices/emergencysituations-medical-devices/emergency-use-authorizations

4. Accula test: SARS-CoV-2 test. U.S. Food \& Drug Administration https://www.fda.gov/media/136355/download (2020).

5. Abbott realtime SARS-CoV-2 assay. Abbott https://www. molecular.abbott/us/en/products/infectious-disease/ RealTime-SARS-CoV-2-Assay (2020).

6. SARS-CoV-2 Diagnostic Pipeline (Find, 2020); https://www.finddx. org/covid-19/pipeline/

7. COVID-19 Coronavirus rapid test casette. SureScreen Diagnostics https://www.surescreen.com/products/covid-19-corona virus-rapid-test-cassette (2020).

8. Parra, A., Giles, C. \& Lawless, J. Virus test results in minutes? Scientists question accuracy. Associated Press News https:// apnews.com/94d68c23955a4d7222f6b9cb47ea89cc (2020).

9. $\mathrm{Hu}, \mathrm{K}$. et al. Preprint at https://doi. org/10.1101/2020.03.19.20026245 (2020).

10. Verity, R. et al. Lancet Infect. Dis. https://doi.org/10.1016/S14733099(20)30243-7 (2020).

11. Today's Data on the Geographic Distribution of COVID-19 Cases Worldwide (European Centre for Disease Prevention and Control, 2020); https://www.ecdc.europa.eu/en/publicationsdata/download-todays-data-geographic-distribution-covid19-cases-worldwide

12. Roser, M., Ritchie, H. \& Ortiz-Ospina, E. Coronavirus Disease (COVID-19) - Statistics and Research (Our World in Data, 2020); https://ourworldindata.org/coronavirus

13. Pàmies, P. Tracking COVID-19 cases and deaths. Nature Research Bioengineering Community https://bioengineeringcommunity. nature.com/users/20986-pep-pamies/posts/64985-tracking-covid19-cases-and-deaths (2020). 